\title{
Efficacy and Safety of Sodium Hyaluronate with 1,4-Butanediol Diglycidyl Ether Compared to Sodium Carboxymethylcellulose in Preventing Adhesion Formation after Lumbar Discectomy
}

\author{
Gyu Yeul $\mathrm{Ji}^{1,2^{*}}$, Chang Hyun $\mathrm{Oh}^{2^{*}}$, Byung Gwan Moon ${ }^{3}$, Seong $\mathrm{Yi}^{1}$, In Bo Han ${ }^{4}$, \\ Dong Hwa $\mathrm{Heo}^{5}$, Ki-Tack Kim ${ }^{6}$, Dong Ah Shin ${ }^{1}$, Keung Nyun Kim ${ }^{1}$ \\ ${ }^{I}$ Department of Neurosurgery, Yonsei University College of Medicine, Seoul, \\ ${ }^{2}$ Department of Neurosurgery, Guro Teun Teun Hospital, Seoul, \\ ${ }^{3}$ Department of Neurosurgery, Eulji Hospital, Eulji University College of Medicine, Seoul, \\ ${ }^{4}$ Department of Neurosurgery, Bundang CHA Medical Center, CHA University College of Medicine, Sungnam, \\ ${ }^{5}$ Department of Neurosurgery, The Leon Wiltse Memorial Hospital, Suwon, \\ ${ }^{6}$ Department of Orthopedic Surgery, Kyung Hee University College of Medicine, Seoul, Korea
}

Objective: Epidural injection of hyaluronic acid may prevent adhesion formation after spine surgery, but the compounds used to stabilize hyaluronidase could interfere with its anti-adhesion effects. The present study was conducted as a clinical trial to evaluate the efficacy and safety of an experimental medical gel in preventing adhesion formation.

Methods: This study was designed as a multicenter, randomized, double-blind, and comparative controlled clinical trial with an observation period of 6 weeks. Subjects were randomly assigned into two groups: group A with sodium hyaluronate + 1,4-butanediol diglycidyl ether (BDDE) and group B with sodium hyaluronate + sodium carboxymethylcellulose (CMC). Visual analogue scale (VAS) of back and leg pain and the Oswestry disability index (ODI) and scar score ratings were assessed after surgery.

Results: Mean scar grade was $2.37 \pm 1.13$ in group $A$ and $2.75 \pm 0.97$ in group $B$, a statistically significant difference $(p=0.012)$. VAS of back and leg pain and ODI scores decreased significantly from baseline to 3 and 6 weeks postoperatively in both groups $(p<0.001)$. However, VAS and ODI scores were not statistically different between groups A and B at baseline or at 3 and 6 weeks after operation $(p>0.3)$. The number of adverse reactions related to the anti-adhesion gels was not statistically different $(p=0.569)$, but subsequent analysis of nervous adverse reactions showed group $B$ was superior with a statistically difference $(p=0.027)$.

Conclusion: Sodium hyaluronate with BDDE demonstrated similar anti-adhesion properties to sodium hyaluronate with CMC. But, care should be used to nervous adverse reactions by using sodium hyaluronate with BDDE.

Key Words: Anti-adhesion • Scar formation • Lumbar discectomy • Sodium hyaluronate $•$ 1,4-butanediol diglycidyl ether (BDDE) • Sodium carboxymethylcellulose (CMC)

\section{INTRODUCTION}

Failed back surgery syndrome (FBSS) is defined as the presence of persistent or severe disabling pain that continues after spinal surgery ${ }^{2,23,25)}$. According to previous studies, surgical

treatment of intervertebral disc herniation showed relatively good results in $90 \%$ of patients, but failed in $10 \%{ }^{17,26}$. Epidural fibrosis, which can be occurred in the course of surgical treatment, may be the cause of such poor results ${ }^{5}$, and in previous studies, was observed in $24 \%$ of recently failed lumbar spine surgeries ${ }^{19,32)}$. Moreover, the degree of back pain or nerve root pain could be reflective of the extent of spinal epidural

- Received: December 9, 2014 - Revised: March 30, 2015 • Accepted: April 1, 2015

Corresponding Author: Dong Ah Shin, MD, PhD

Department of Neurosurgery, Yonsei University College of Medicine, 50-1, Yonsei-ro, Seodaemun-gu, Seoul 120-752, Korea

Tel: +82-2-2228-2150, Fax: +82-2-393-9979, E-mail: shindongah@me.com

*These authors contributed equally in this article, so should be considered as co-first authors.

"This study has not been presented in part elsewhere.

@This is an Open Access article distributed under the terms of the Creative Commons Attribution Non-Commercial License (http://creativecommons.org/

licenses/by-nc/3.0/) which permits unrestricted non-commercial use, distribution, and reproduction in any medium, provided the original work is properly cited. 
fibrosis $^{21,27)}$. Recently, various surgical materials prepared via polymer synthesis from natural resources to prevent or reduce adhesions after surgery have been developed ${ }^{12,18,28,31,32)}$. One such substance, hyaluronidase, has been administered to reduce swelling and edema in tissues by dissolving the glucosaminic bonds between hyaluronic acid and connective tissue. Utilizing this process, epidural injection of hyaluronic acid may prevent adhesion formation. However, the compounds used to stabilize hyaluronidase could interfere with its anti-adhesion effects. Previously, unpublished data from an effect test report of sodium hyaluronate +1 ,4-butanediol diglycidyl ether (BDDE) demonstrated a greater anti-adhesion effect for BDDE than for other compounds of sodium hyaluronate. Here the authors conducted a clinical trial of an experimental medical gel composed of hyaluronate + BDDE to evaluate its effectiveness and safety in preventing adhesion formation.

\section{MATERIALS AND METHODS}

This clinical trial was designed as a multicenter, randomized, double-blind, and comparative controlled clinical trial. All subjects were volunteers and provided signed informed consent. In total, 74 cases that satisfied the selection criteria were enrolled for the study period of 24 months, and 68 cases were included in the final analyses, as 6 cases dropped out during the observation period (6 weeks for each case). The subjects were randomly divided into one of two groups according to type of anti-adhesion agent to be applied: group A received the anti-adhesion gel HyFence $\mathrm{LV}^{\circledR}$ (sodium hyaluronate + 1,4-Butanediol diglycidyl ether (BDDE), Cha Bio \& Diostech, Seoul, Republic of Korea) and group B was treated with Guardix-SOL ${ }^{\circledR}$ (sodium hyaluronate + sodium carboxymethylcellulose, Genewel Co, Seongnam, Republic of Korea). All subjects received a randomly assigned anti-adhesion gel before wound closure after standard discectomy. Clinical outcomes were evaluated according to visual analogue scale (VAS) assessment and the Korean version of the Oswestry Disability Index (ODI) before surgery as well as 3 and 6 weeks after the investigational medical agents had been applied. Radiological outcomes were evaluated by magnetic resonance imaging (MRI), which was performed at 6 weeks after surgery, and graded according to scar formation by three different independent evaluators blinded to information about the subjects. Scar score ratings were graded according to the degree of dural scar score in the spinal canal as presented in Fig. 1 and Table 1. For each subject, five cross-sectional MR images were checked, and each cross-sectional image was divided into four quadrants, so that a total of 20 quadrant cross-sectional MR images per subject were evaluated ${ }^{27)}$. The checked scar grades of 3 different observers were all categorized by the scar grades and the mean scar grade was calculated.

The inclusion criteria for this study consisted of (1) patients older than 20 years and younger than 65 years of age; (2) diagnosis of lumbar disc herniation at spinal level L4-L5 or L5-S1 with radiculopathic symptoms refractory to conservative treatment for a minimum of 2 weeks; (3) scheduled for first surgery to remove single level and unilateral lumbar disc herniation; (4) could understand and follow instructions; and (5) could provide written consent to participate in the clinical trial. The exclusion criteria consisted of (1) patients with multi-level, far lateral or bilateral lumbar disc herniation; (2) those with degenerative spinal cord disease or scoliosis; (3) exhibited hyaluronic acid-sensitive side effects; (4) involved lymphatic or blood clotting disorders or were administered a blood coagulant; (5) had uncontrolled diabetes; (6) received oral steroid medication within 4 weeks, epidural steroid injection within 10 days or were administered aspirin and/or non-steroidal anti-inflammatory agents within 7 days from the baseline study period; (7) had collagen-vascular disease, self-immune diseases such as rheumatoid arthritis or systemic lupus erythematosus, or malignant tumors within 5 years; (8) demonstrated spinal cord angiography or lumbar puncture within 24 hours from the baseline study period; (9) showed reduced immunity or abnormal labo-
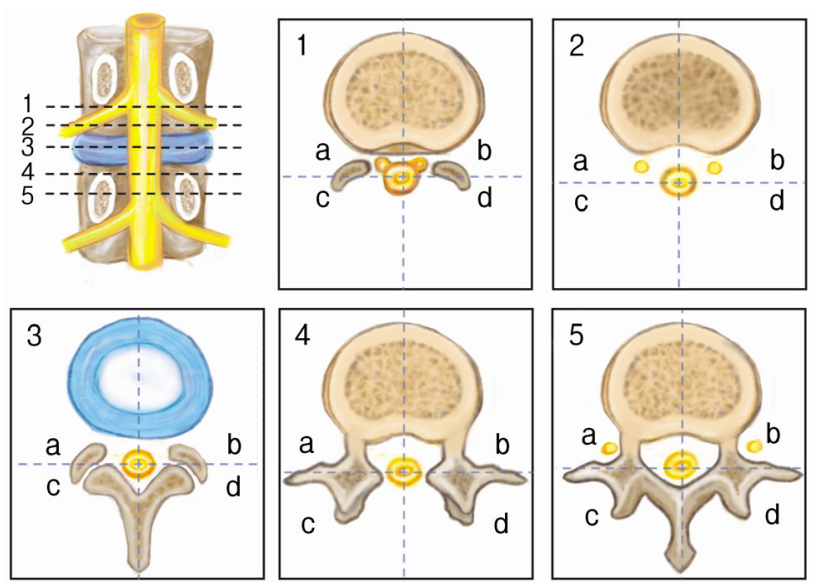

Fig. 1. The positions of five standard cross-sectional magnetic resonance images.

Table 1. Peridural scar score

\begin{tabular}{cc}
\hline \hline Scar score & Description \\
\hline 0 & No/trace scar \\
1 & $0-25 \%$ of quadrant filled with scar \\
2 & $25-50 \%$ of quadrant filled with scar \\
3 & $50-75 \%$ of quadrant filled with scar \\
4 & $>75 \%$ of quadrant filled with scar \\
\hline
\end{tabular}


ratory tests (hematologic, blood chemical, and urine test) during screening tests; (10) have had or currently had serious disability affecting the cardiovascular, digestive, respiratory, endocrine, or central nervous system, or clinical mental illness; (11) participated in other clinical trials within 30 days; or (12) were pregnant, lactating, or on contraceptive medication.

Student's t-test and ANOVA test were conducted to estimate the reliability of radiological and clinical outcomes for each group. Kruskal-Wallis test was used to compare outcomes before application and at follow-up. The mean scar grade of each group was considered as interval scales for easier comparison between the groups, although this should be considered as ordinal scales. All statistical analyses were performed using SPSS software version 12 (SPSS Inc., Chicago, IL, USA), and statistical significance was defined as $\mathrm{p}<0.05$.

\section{RESULTS}

The results concerning scar formation are presented in Table 1. No statistical difference was observed in the demographic data between groups A and B. Scar scores differed according to the different anti-adhesion gels applied, and comprised 3 cases of grade 0,21 cases of grade 1, 35 cases of grade 2, 21 cases of grade 3, and 22 cases of grade 4 scarring in group A. Group B comprised no cases of grade 0,9 cases of grade 1, 37 cases of grade 2, 27 cases of grade 3, and 29 cases of grade 4 scarring. Statistical analysis using the chisquare test generated a result of 0.048 . The mean scar grade for each group was $2.37 \pm 1.13$ in group A and $2.75 \pm 0.97$ in group $\mathrm{B}$, which was a statistically significant difference $(p=0.012)$ (Table 2).

In group A, mean back pain VAS decreased significantly $(\mathrm{p}<0.001)$ from a baseline of $51.94 \pm 48.72$ to a 3 -week postoperative score of $20.28 \pm 20.57$ and a 6 -week postoperative score of $21.35 \pm 22.77$. Similarly, in group B, preoperative

Table 2. Scar scores between the two groups

\begin{tabular}{lccc}
\hline & Group A & Group B & p-value \\
\hline Device & HyFence LV & Guardix-SOL & \\
Composition & HA+CMC & HA+BDDE & \\
N & 34 & 34 & \\
Grade 0 & 3 & 0 & 0.048 \\
Grade 1 & 21 & 9 & \\
Grade 2 & 35 & 37 & \\
Grade 3 & 21 & 27 & \\
Grade 4 & 22 & 29 & \\
Mean Grade & $2.37 \pm 1.13$ & $2.75 \pm 0.97$ & 0.012
\end{tabular}

$\mathrm{HA}$, sodium hyaluronate; $\mathrm{CMC}$, sodium carboxymethylcellulose; BDDE, 1,4-Butanediol diglycidyl ether mean back pain VAS score decreased significantly $(\mathrm{p}<0.001)$ from $48.72 \pm 27.87$ to $25.24 \pm 24.45$ at 3 weeks postoperative and $20.41 \pm 21.97$ at 6 weeks postoperative. Mean leg pain VAS significantly decreased $(\mathrm{p}<0.001)$ from a baseline of $63.47 \pm 22.79$ to a 3 -weeks postoperative score of $23.22 \pm$ 23.75 and 6-week postoperative score of $20.59 \pm 26.05$ in group A, and similarly, from a preoperative score of $66.59 \pm$ 20.11 to a 3-week postoperative score of $28.76 \pm 27.30$ and a 6-week postoperative score of $23.32 \pm 26.92$ in group B $(\mathrm{p}<0.001)$. Similar to the VAS results, in group A, mean ODI score decreased significantly $(\mathrm{p}<0.001)$ from a baseline score of $42.11 \pm 16.89 \%$ to $27.93 \pm 14.92 \%$ at 3 weeks postoperative and $22.64 \pm 14.87 \%$ at 6 weeks postoperative. In group B, mean preoperative ODI score decreased from $44.72 \pm 19.11 \%$ to $31.26 \pm 15.41 \%$ at 3 weeks postoperative and $26.62 \pm 16.98$ $\%$ at 6 weeks postoperative (Fig. 2). Altogether, back pain VAS, leg pain VAS and ODI scores were not statistically different between the two groups at baseline or at 3 and 6 weeks after operation $(\mathrm{p}>0.3)$.

Observed adverse reactions are summarized in Table 3. Adverse reactions were categorized as musculoskeletal and connective tissue disorders; injury, poisoning and procedural complications; gastrointestinal disorders; nervous system disorders; general disorders and administration site conditions; renal and urinary disorders; infections and infestations; investigations; psychiatric disorders; skin and subcutaneous tissue disorders; respiratory, thoracic and mediastinal disorders; metabolism and nutrition disorders; hepatobiliary disorders; or vascular disorders. Relevant disease or symptoms for each category are listed in Table 3. In group A, 113 events of an adverse reaction among 33 cases (91.7\%) were recorded, while 98 events among 24 cases (68.6\%) were observed in group B. Although the number of cases of an adverse reaction between the two groups was statistically different $(p=0.018)$, the number of adverse reactions was not statistically different $(p=$ 0.569). Most of the adverse reactions were minor (two major adverse reactions in group B included one case of wound infection and a case of intervertebral disc protrusion), and all adverse reactions were unrelated to the device. In regards to adverse reaction category, nervous system disorders were more frequently recorded for group A than group B; 14 events were recorded among 12 patients in group $\mathrm{A}$, while 7 events among 4 patients were noted in group $B(p=0.027)$. But nervous adverse reactions broadly included symptoms of paraesthesia, dizziness, hypoesthesia, carpal tunnel syndrome, cerebral arteriosclerosis, cervicobrachial syndrome, headache and radicular pain. Concerning the presenting symptoms of the adverse nervous system disorders, five events of paraesthesia in group A and one event in group B were observed; one event of dizziness in group A and 4 events in group B were recorded; 4 events 

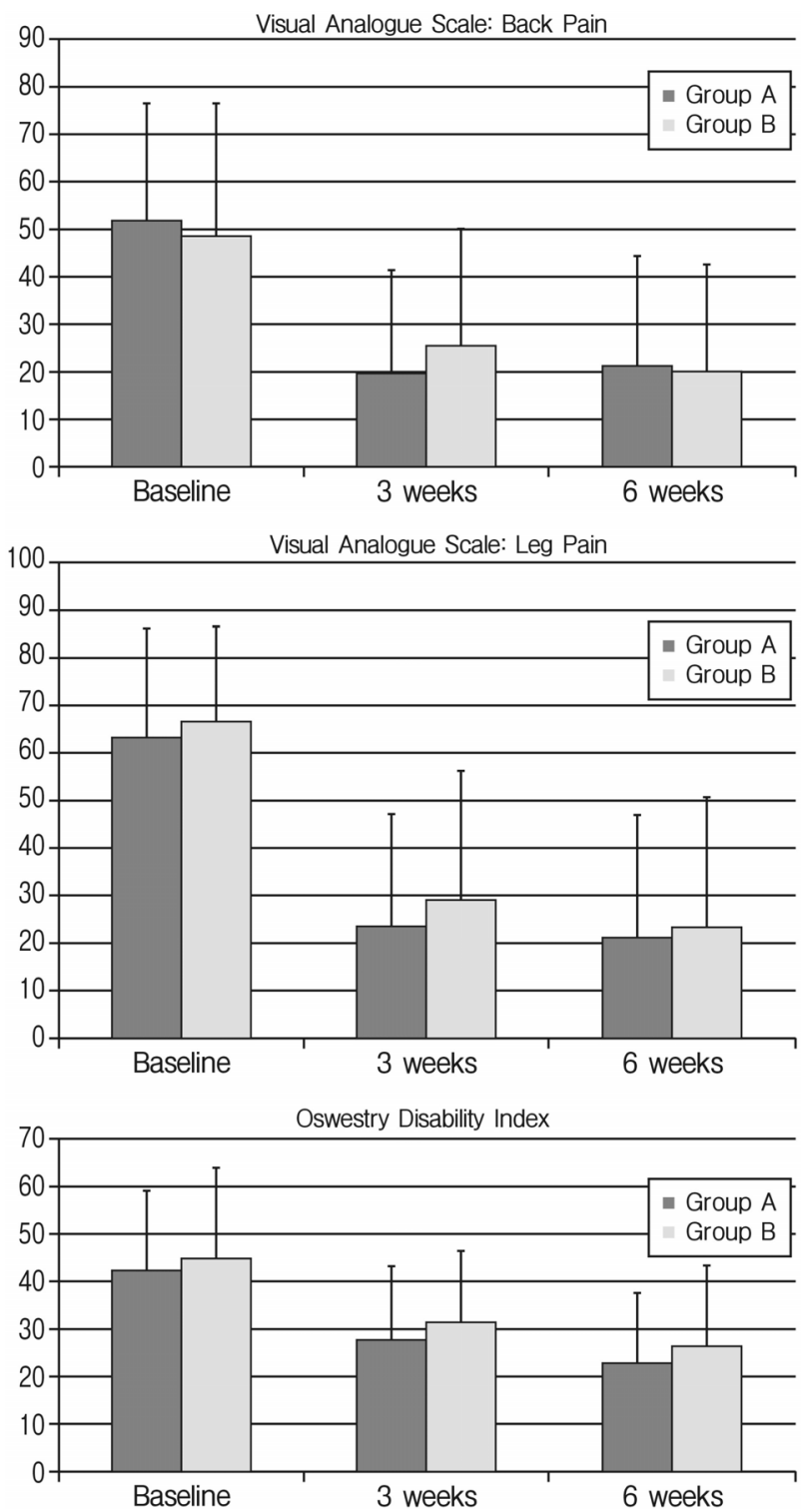

Fig. 2. Clinical results between the two groups: visual analog scale for back and leg pain, and Oswestry disability index.

of hypoesthesia in group A and one event in group B were noted; one event each of carpal tunnel syndrome, cerebral arteriosclerosis, cervicobrachial syndrome and radicular pain were recorded in group A; and finally, a single event of headache was reported in group B. Subsequent analysis of nervous adverse reactions that could related to the anti-adhesion gels (e.g. paraesthesia, hypoesthesia and radicular pain) showed a statistically difference $(p=0.013)$. In other adverse reaction categories, no statistical difference was observed.
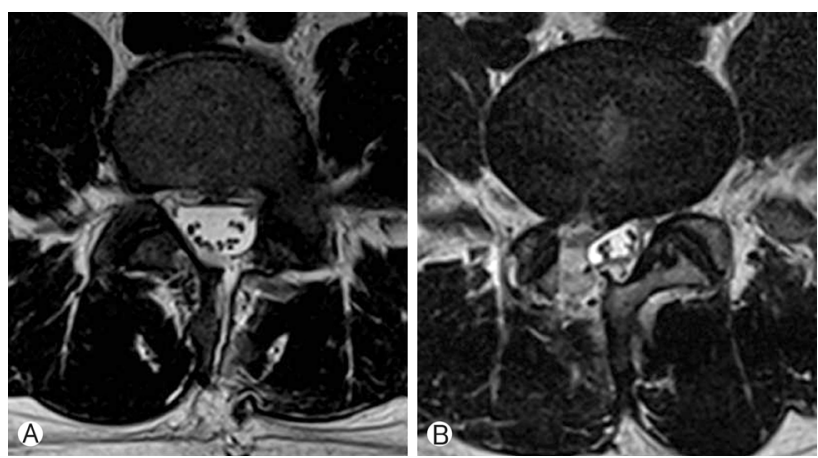

Fig. 3. Postoperative MR. (A) Scantly observed scar tissue; and (B) Compressive scar tissue in post-laminectomy site.

\section{DISCUSSION}

Postoperative fibrosis is a natural course of wound healing ${ }^{12,24)}$. Fibroblasts, originating from the overlying muscles and following extension of postoperative hematoma into the vertebral canal, release excessive extracellular matrix and cause the abundance and strong adhesion of the tissue ${ }^{16,29)}$. Thus, the migration of fibroblasts from the raw surface of the erector spine musculature was stated as the source of postoperative scar tissue $e^{10,16}$. But, epidural adhesion was considered as a major contributing factor to postsurgery radicular pain and lower extremity weakness after laminectomy ${ }^{3,7,11,18,19,22,24,27)}$. Moreover, epidural adhesion is thought to be with the increased complication rates associated with spinal reinterventions ${ }^{4,6,9,13)}$. A general feature seems to be the requirement for direct contact between exposed dura and invading fibroblasts, thereby allowing for the generation of localized dense fibrotic tissue and tethering of the thecal sac and nerve roots ${ }^{24)}$. Numerous experimental and clinical studies had focused to prevent epidural adhesion formation using prophylactic intervention. Such treatments have included modified surgical approaches, antiinflammatory agents, antibiotics, and a wide variety of biological and synthetic barriers, including fat grafts, hyaluronan, collagen, gelatin foam, polylactide films, ADCON-L and more recently, Oxiplex1/SP Gel. ${ }^{1,6,8,11,13-15,20,21,24,27,33)}$. In general concept, the ideal agent for preventing peridural adhesion and fibrosis should include the following properties: (1) prevention of scar tissue adhesion to the dural tissues, (2) prevention of the development of leptomeningeal arachnoiditis, (3) no potential to impair dural healing following tearing and CSF leakage and (4) no capability to induce excessive inflammation around neural tissues ${ }^{24)}$.

In the present study, scar scores were significantly different between the two medical agents. The mean scar grade for group A was $2.46 \pm 0.71$ and $2.70 \pm 0.71$ for group B, a statistically 
Table 3. Adverse effects observed between the two groups

\begin{tabular}{|c|c|c|c|}
\hline & Group A (n=36) & Group B (n=35) & p-value \\
\hline Cases of adverse reactions & $n=33(91.7 \%)$ & $n=24(68.6 \%)$ & 0.018 \\
\hline Events of adverse reactions & 113 & 98 & 0.569 \\
\hline Major adverse reactions & 0 & 2 & \\
\hline Minor adverse reactions & 113 & 96 & 0.146 \\
\hline Device related adverse reactions & 0 & 0 & \\
\hline Non-device related adverse reactions & 113 & 98 & N/A \\
\hline \multicolumn{4}{|l|}{ Adverse Reactions } \\
\hline Musculoskeletal and connective tissue disorders ${ }^{1}$ & $23(n=12,33.3 \%)$ & $18(n=9,25.7 \%)$ & 0.482 \\
\hline Injury, poisoning and procedural complications ${ }^{2}$ & $21(n=16,44.4 \%)$ & $18(n=12,34.3 \%)$ & 0.381 \\
\hline Gastrointestinal disorders ${ }^{3}$ & $20(n=16,44.4 \%)$ & $18(n=12,34.3 \%)$ & 0.381 \\
\hline Nervous system disorders ${ }^{4}$ & $14(n=12,33.3 \%)$ & $7(n=4,11.4 \%)$ & 0.027 \\
\hline General disorders and administration site conditions ${ }^{5}$ & $8(n=8,22.2 \%)$ & $9(n=8,22.9 \%)$ & 0.949 \\
\hline Renal and urinary disorders ${ }^{6}$ & $7(n=7,19.4 \%)$ & $7(n=7,20.0 \%)$ & 0.953 \\
\hline Infections and infestations ${ }^{7}$ & $5(n=3,8.3 \%)$ & $6(n=6,17.1 \%)$ & 0.265 \\
\hline Investigations ${ }^{8}$ & $5(n=4,11.1 \%)$ & $4(n=4,11.4 \%)$ & 0.966 \\
\hline Psychiatric disorders ${ }^{9}$ & $4(n=4,11.1 \%)$ & $2(n=2,5.7 \%)$ & 0.413 \\
\hline Skin and subcutaneous tissue disorders ${ }^{10}$ & $3(n=2,5.6 \%)$ & $3(n=3,8.6 \%)$ & 0.620 \\
\hline Respiratory, thoracic and mediastinal disorders ${ }^{11}$ & $2(n=2,5.6 \%)$ & $2(n=1,2.9 \%)$ & 0.572 \\
\hline Metabolism and nutrition disorders ${ }^{12}$ & $0(n=0,0.0 \%)$ & $2(n=2,5.7 \%)$ & 0.146 \\
\hline Hepatobiliary disorders ${ }^{13}$ & $0(n=0,0.0 \%)$ & $2(n=1,2.9 \%)$ & 0.307 \\
\hline Vascular disorders ${ }^{14}$ & $1(n=1,2.8 \%)$ & $0(n=0,0.0 \%)$ & 0.321 \\
\hline
\end{tabular}

${ }^{1}$ Back pain, pain in an extremity, musculoskeletal pain, intervertebral disc protrusion, muscular weakness, arthralgia, musculoskeletal stiffness, myalgia, and myofascial pain syndrome; ${ }^{2}$ Procedural pain, post procedural complication, procedural hypertension, wound secretion, contusion, open wound, post procedural constipation, post procedural discharge, post procedural discomfort, post procedural edema, post procedural swelling, procedural nausea, and toxicity to various agents; ${ }^{3}$ Constipation, nausea, dyspepsia, vomiting, aphthous stomatitis, diarrhea, gastrointestinal disorder, abdominal distension, abdominal pain, dry mouth, hematochezia, and reflux esophagitis; ${ }^{4}$ Paraesthesia, dizziness, hypoesthesia, carpal tunnel syndrome, cerebral arteriosclerosis, cervicobrachial syndrome, headache, and radicular pain; ${ }^{5}$ Pyrexia, chills, hernia, pain, and tenderness; ${ }^{6}$ Dysuria and urinary retention; ${ }^{7}$ Nasopharyngitis, gastroenteritis, upper respiratory tract infection, and wound infection; ${ }^{8}$ Increased blood pressure, increased alanine aminotransferase, and increased aspartate aminotransferase; 'Insomnia, anxiety, and depression; ${ }^{10}$ Unticaria, dermatitis contact, pruritus, and rash; ${ }^{11}$ Atelectasis, cough, dyspnea, and oropharyngeal pain; ${ }^{12}$ Decreased appetite and hypoalbuminemia; ${ }^{13}$ Hepatic cyst and hepatic steatosis; ${ }^{14}$ Hypertension

significant difference $(\mathrm{p}=0.05)$. Moreover, all back pain VAS, leg pain VAS and ODI scores decreased significantly $(\mathrm{p}<$ 0.001 ) from baseline to 3 and 6 weeks postoperative. These results indicated that 1,4-butanediol diglycidyl ether is a more effective compound than carboxymethyl cellulose in stabilizing sodium hyaluronate, and substantiated our results from a previous, unpublished preliminary study in rats. Although no absolute proof has been presented and the invasion was needed, the authors discerned that 1,4-butanediol diglycidyl ether is a more effective stabilizer of sodium hyaluronate after spine operation as a result of its molecular structure and other unknown variables. However, interestingly, although all of the adverse reactions observed in this study were not related to the agent, nervous system disorders were more frequently reported in 1,4-butanediol diglycidyl ether administered sub- jects with statistical significance $(p=0.027)$. Indeed, the presenting symptoms were directly correlated with nerve root irritation such as paraesthesia or hypoesthesia. Therefore, careful patient selection in the use of sodium hyaluronate with 1,4-butanediol diglycidyl ether is needed and more specific study concerning this issue is warranted.

There were some limitations of this study that warrant consideration. First, this study was initially designed as a noninferiority test of sodium hyaluronate with 1,4-butanediol diglycidyl ether compared the other anti-adhesive products; therefore, further compatible randomized control study is needed. Second, scar formation was checked not by clinical study, but by imaging studies. Therefore, the actual scar formation is not clear. Indeed, the postoperative peridural scar was commonly studied after postoperative 6 months, but this study was evalua- 
ted at 6 weeks after operation. So, the MR finding is susbjective postoperative epidural scar, but, it could be misjudge of the epidural soft tissue edema or hemorrhage. So, further elongated study is needed to confirm the scar formation by the adhesion formation materials. Despite these limitations, the present study was designed as a multicenter, randomized, single-blind, and comparative controlled clinical trial. The results indicated that stabilizing materials of sodium hyaluronate interfere with the anti-adhesive function thereof after spine surgery and that 1,4-butanediol diglycidyl ether is a more effective compound than carboxymethylcellulose in stabilizing sodium hyaluronate.

\section{CONCLUSION}

Sodium hyaluronate with 1,4-butanediol diglycidyl ether demonstrated better anti-adhesion properties than sodium hyaluronate with carboxymethylcellulose in patients after lumbar discectomy. However, further research could help to know its ambiguous effect on the neural tissues.

\section{ACKNOWLEDGMENT}

This study was received the external funding from Cha Bio \& Diostech, and the authors declare that they have no proprietary, commercial, or financial interests that could be construed to have inappropriately influenced this study.

\section{REFERENCES}

1. Akeson WH, Massie JB, Huang B, Giurea A, Sah R, Garfin SR, et al: Topical high-molecular-weight hyaluronan and a roofing barrier sheet equally inhibit postlaminectomy fibrosis. Spine J 5:180-190, 2005

2. Asch HL, Lewis PJ, Moreland DB, Egnatchik JG, Yu YJ, Clabeaux DE, et al: Prospective multiple outcomes study of outpatient lumbar microdiscectomy: should 75 to $80 \%$ success rates be the norm? J Neurosurg 96:34-44, 2002

3. BenDebba M, Augustus van Alphen H, Long DM: Association between peridural scar and activity-related pain after lumbar discectomy. Neurol Res 21:S37-42, 1999

4. Benoist M, Ficat C, Baraf P, Cauchoix J: Postoperative lumbar epiduro-arachnoiditis. Diagnostic and therapeutic aspects. Spine 5:432-436, 1980

5. Burton CV, Kirkaldy-Willis WH, Yong-Hing K, Heithoff KB: Causes of failure of surgery on the lumbar spine. Clin Orthop Relat Res 157:191-199, 1981

6. de Tribolet N, Porchet F, Lutz TW, Gratzi O, Brotchi J, van Alphen HA, et al: Clinical assessment of a novel antiadhesion barrier gel: Prospective, randomized, multicenter, clinical trial of ADCON-L to inhibit postoperative peridural fibrosis and related symptoms after lumbar discectomy. Am J Orthop 27: 111-120, 1998

7. Gambardella G, Gervasio O, Zaccone C, Puglisi E: Prevention of recurrent radicular pain after lumbar disc surgery: A prospective study. Acta Neurochir 92:151-154, 2005

8. Hinton JL Jr, Warejcka DJ, Mei Y, McLendon RE, Laurencin C, Lucas PA, et al: Inhibition of epidural scar formation after laminectomy in the rat. Spine 20:564-570, 1995

9. Kayaoglu CR, Calikoglu C, Binler S: Re-operation after lumbar disc surgery: Results in 85 cases. J Int Med Res 31:318-323, 2003

10. Kemaloglu S, Ozkan U, Yilmaz F, Nas K, Gur A, Acemoglu $\mathrm{H}$, et al: Prevention of spinal epidural fibrosis by recombinant tissue plasminogen activator in rats. Spinal Cord 41:427-431, 2003

11. Kim KD, Wang JC, Robertson DP, Brodke DS, Olson EM, Duberg AC, et al: Reduction of radiculopathy and pain with Oxiplex/SP Gel after laminectomy, laminotomy and discectomy: A pilot clinical study. Spine 28:1080-1087, 2003

12. Klopp LS, Welch WC, Tai JW, Toth JM, Cornwall GB, Turner AS: Use of polylactide resorbable film as a barrier to postoperative peridural adhesion in an ovine dorsal laminectomy model. Neurosurg Focus 16:E2, 2004

13. Kuivila TE, Berry JL, Bell GR, Steffee AD: Heparinized materials for control of the formation of the laminectomy membrane in experimental laminectomies in dogs. Clin Orthop Relat Res 236: 166-174, 1988

14. Lee JY, Stenzel W, Ebel H, Wedekind C, Ernestus RI, Klug $\mathrm{N}$ : Mitomycin $\mathrm{C}$ in preventing spinal epidural fibrosis in a laminectomy model in rats. J Neurosurg Spine 100:52-55, 2004

15. Lee JY, Stenzel W, Impekoven P, Theisohn M, Stützer H, Löhr $\mathrm{M}$, et al: The effect of mitomycin $\mathrm{C}$ in reducing epidural fibrosis after lumbar laminectomy in rats. J Neurosurg Spine 5:53-60, 2006

16. Liu J, Ni B, Zhu L, Yang J, Cao X, Zhou W: Mitomycin Cpolyethylene glycol controlled-release film inhibits collagen secretion and induces apoptosis of fibroblasts in the early wound of a postlaminectomy rat model. Spine J 10:441-447, 2010

17. Loupasis GA, Stamos K, Katonis PG, Sapkas G, Korres DS, Hartofilakidis G: Seven- to 20- year outcome of lumbar discectomy. Spine 24:2313-2317, 1999

18. QManchikanti L, Saini B, Singh V: Spinal endoscopy and lysis of epidural adhesions in the management of chronic low back pain. Pain Physician 3:240-265, 2001

19. Maroon JC, Abla A, Bost J: Association between peridural scar and persistent low back pain after lumbar discectomy. Neurol Res 21:S43-46, 1999

20. Nakano M, Matsui H, Miaki K, Tsuji H: Postlaminectomy adhesion of the cauda equina. Inhibitory effects of antiinflammatory drugs on cauda equina adhesion in rats. Spine 23:298- 304, 1998

21. Ozer AF, Oktenoglu T, Sasani M, Bozkus H, Canbulat N, Karaarslan E, et al: Preserving the ligamentum flavum in lumbar disce ctomy: a new technique that prevents scar tissue formation in the first 6 months postsurgery. Neurosurgery 59:126-133, 2006

22. Parke WW, Watanabe R: Adhesions of the ventral lumbar dura. An adjunct source of discogenic pain? Spine 15:300-303, 1990 23. Pheasant HC, Dyck P: Failed lumbar disc surgery: cause, assess- 
ment, treatment. Clin Orthop 164:93-109, 1982

24. Richards PJ, Turner AS, Gisler SM, Kraft S, Nuss K, Mark S, et al: Reduction in postlaminectomy epidural adhesions in sheep using a fibrin sealant-based medicated adhesion barrier. J Biomed Mater Res B Appl Biomater 92:439-446, 2010

25. Robertson JT: Role of peridural fibrosis in the failed back: a review. Eur Spine J 5:S2-6, 1996

26. Rönnberg K, Lind B, Zoega B, Gadeholt-Göthlin G, Halldin K, Gellerstedt M, et al: Peridural scar and its relation to clinical outcome: a randomized study on surgically treated lumbar disc herniation patients. Eur Spine J 17:1714-1720, 2008

27. Ross JS, Robertson JT, Frederickson RC, Petrie JL, Obuchowski $\mathrm{N}$, Modic MT, et al: Association between peridural scar and recurrent radicular pain after lumbar discectomy: Magnetic resonance evaluation.: ADCON-L European Study Group. Neurosurgery 8:855-863, 1996

28. Schimizzi AL, Massie JB, Murphy M, Perry A, Kim CW, Garfin SR, et al: High-molecular-weight hyaluronan inhibits macrophage proliferation and cytokine release in the early wound of a preclinical postlaminectomy rat model. Spine J 6:550-556, 2006

29. Songer MN, Rauschning W, Carson EW, Pandit SM: Analysis of peridural scar formation and its prevention after lumbar laminotomy and discectomy in dogs. Spine 20:571-580, 1995

30. Spangfort EV: The lumbar disc herniation: A computer-aided analysis of 2, 504 operations. Acta Orthop Scand Suppl 142:195, 1972

31. Tatsui CE, Martinez G, Li X, Pattany P, Levi AD: Evaluation of DuraGen in preventing peridural fibrosis in rabbits. Invited submission from the joint section meeting on disorders of the spine and peripheral nerves. J Neurosurg Spine 4:51-59, 2006

32. Vakis A, Koutentakis D, Karabetsos D, Kalostos G: Use of polytetrafluoroethylene dural substitute as adhesion preventive material during craniectomies. Clin Neurol Neurosurg 108:798-802, 2006

33. Yucesoy K, Karci A, Kilicalp A, Mertol T: The barrier effect of laminae: Laminotomy versus laminectomy. Spinal Cord 38:442444, 2000 